\title{
Current dietetic practices in the management of gestational diabetes mellitus: a survey of Malaysian dietitians
}

\begin{abstract}
The role of the dietitian in the management of Gestational Diabetes Mellitus (GDM) is highly recognised. However, consensuses on dietetic approaches for the management of GDM in Malaysia are scarce. This study aimed to examine the current dietetic practices in the management of GDM and to compare nutrient recommendations provided by dietitians to those of several established international guidelines. A survey was conducted among dietitians who were working in government affiliated hospitals across Malaysia. Of 148 questionnaires posted to dietitians, a total of 101 were returned representing an overall response rate of $68 \%$. The majority of the dietitians $(80 \%)$ reported that they see women with GDM in their daily practices with more than half (53\%) having counselled 5 to 9 women per day during the previous 3 months. Flexible carbohydrate exchanges (82\%) was the most common recommendation on carbohydrate intake followed by advice regarding small frequent meals spread over the day $(62 \%)$ and portion control by using the plate method (54\%). Only $11 \%$ dietitians incorporated the use of the glycaemic index as a measure of carbohydrate intake into their intervention. While recommendations regarding protein intake were consistent with established guidelines, the amount of carbohydrate recommended by the dietitians was higher than that in established guidelines. Although consistency was seen in key components of nutrition intervention, there were differences in the delivery of nutrient recommendations particularly in regard to dietary carbohydrate intake indicating a need for consensus on dietetic practice guidelines for the management of GDM.
\end{abstract}

Keyword: Dietetic practices; Gestational Diabetes Mellitus (GDM); Nutrient recommendations; Malaysia; Dietitians 